\title{
Endoscopic Banding for Duodenal Carcinoid Tumors
}

\author{
Ashwin Rammohan, Sathyanesan Jeswanth, Perumal Senthil Kumar, Palaniappan Ravichandran
}

\section{ABSTRACT}

Introduction: Carcinoids of the duodenum are rare and have an indolent course. Most of these patients have been conventionally subjected to extensive surgery based on the site of the tumor. In this report, we have explored the safety and feasibility of endoscopic banding for such tumors. This to our best knowledge is only the second such report in English medical literature.

Case report In a 50-year-old woman with history of abdominal pain and vomiting, upper gastrointestinal endoscopy revealed a $0.5 \mathrm{~cm}$ umbilicated nodular lesion along the medial wall of the first part of duodenum. Biopsy of the lesion confirmed it to be a carcinoid, and endoscopic ultrasonography (EUS) showed the carcinoid was confined to the submucosa. Endoscopic banding with resection of the lesion was done. Patient tolerated the procedure well and is asymptomatic on follow-up.

Conclusion: Small duodenal carcinoids confined to the submucosa can be safely and effectively managed by endoscopic banding and preoperative. Larger studies in patients with duodenal carcinoids tumors are needed to confirm these promising results.

Keywords: Endoscopic banding, Duodenal carcinoid, Abdominal ultrasonography.

How to cite this article: Rammohan A, J eswanth S, Kumar PS, Ravichandran P. Endoscopic Banding for Duodenal Carcinoid Tumors. Euroasian J Hepato-Gastroenterol 2013; 3(1):78-80.

\section{Source of support $\mathrm{Nil}$}

\section{Conflict of interest: None}

\section{INTRODUCTION}

Carcinoid tumors are the most common primary tumors of the small bowel; they arise from the enterochromaffin cells of Kulchitsky. ${ }^{1}$ Duodenal carcinoids are rare, accounting for only $2 \%$ of such lesions in the small intestine. ${ }^{1,2}$ Even though they have an indolent course, resection is generally recommended due to the risk of lymph node involvement and metastases. ${ }^{1,3} \mathrm{M}$ ost cases of duodenal carcinoid have conventionally been treated by major surgical resections like a duodenal resection or even a W hipple's procedure. ${ }^{1,3,4}$ Recently, endoscopic treatment such as endoscopic mucosal resection (EMR) and endoscopic submucosal dissection (EM D) has been attempted as an al ternative to conventional surgery. ${ }^{3-7}$ The aim of this report is to explore the possibility of endoscopic banding and resection of small duodenal carcinoids, thereby avoiding the morbidity associated with conventional foregut surgery. This report to our knowledge is the only the second such report of endoscopic banding for duodenal carcinoids in English medical literature. ${ }^{8}$

\section{CASE REPORT}

A 50-year-old otherwise healthy woman presented with postprandial upper abdominal pain and intermittent vomiting for the past 5 years. She denied any history of jaundice, hematemesis, melena, flushing of skin or altered bowel habits. She was known to have a history of bronchial asthma for past 40 years. Her general and local examinations were unremarkable apart from minimal epigastric tenderness. Her compl ete hemogram, renal function tests, and liver function tests were within normal limits. Her serum calcitonin levels were also within normal limits. She underwent an upper gastrointestinal endoscopy which showed a nodule along the medial wall of the first part of duodenum (Fig. 1). A biopsy was taken from the nodule which was suggestive of carcinoid tumor. A contrast-enhanced computed tomography (CECT) was taken which showed a mass lesion within the first part of duodenum. No enlarged nodes or space occupying lesions in the liver were identified on CECT. A $n$ endoscopic ultrasound (EUS) was performed which demonstrated a well-defined hypoechoic tumor confined to the submucosal hyperechoic layer with the underlying hypoechoic muscularis propria being intact. Due to the superficial and indolent nature of the carcinoid it was decided to do an endoscopic banding of the lesion. She was optimized and an endoscopic band ligation of the lesion with resection was done. Suction was applied to retract the lesion into the banding device, and a band was deployed to capture the lesion. The banding device was then removed and a standard el ectrocautery snare was used to resect the lesion above the band (Fig. 2). The patient's post-procedure period was uneventful and she was discharged home the

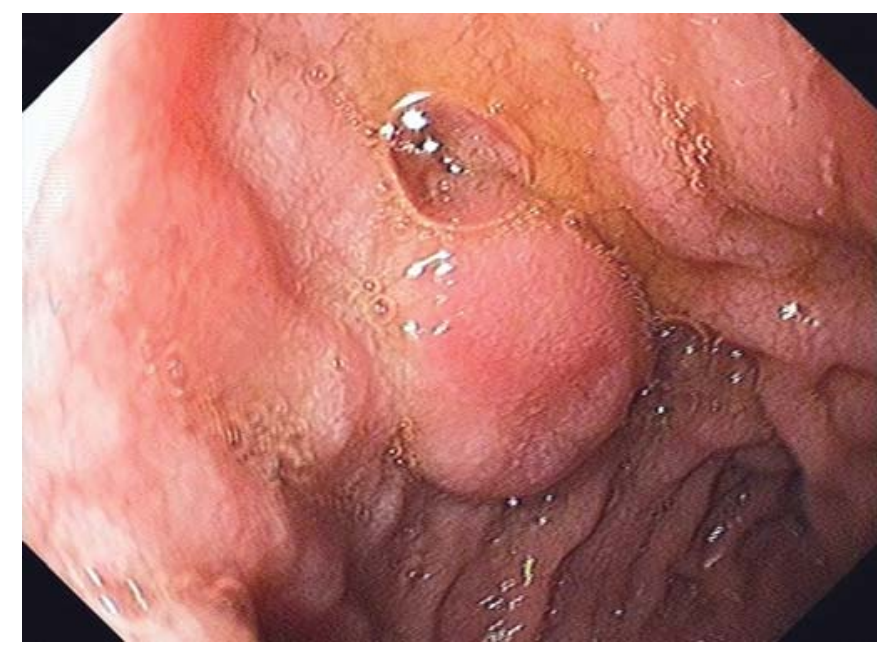

Fig. 1: Carcinoid along the medial wall of first part of duodenum 


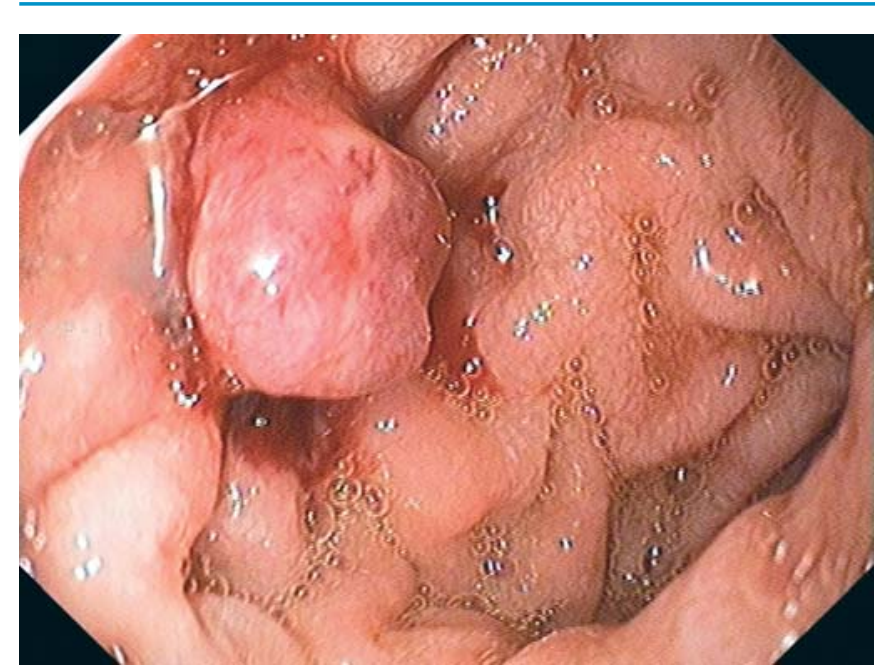

Fig. 2: Duodenal carcinoid postbanding status- preresection

next morning. A repeat outpatient U GI endoscopy at 2 weeks revealed a shallow healing ulcer. She is asymptomatic on follow-up.

\section{DISCUSSION}

Oberndorfer in 1907 first used the term 'karzenoide' to describe a carcinoma like small bowel tumor that behaved in a benign fashion. ${ }^{1}$ Carcinoid tumors usually exhibit a more benign clinical behavior than epithelial carcinomas of the same anatomic site. The term carcinoid represents a diverse group of neoplasms arising from enterochromaffin cells which are believed to be a part of the amine precursor uptake and decarboxylation (APUD) system and serve endocrine functions. ${ }^{1,2}$ D uodenal carcinoids accounting for only $2 \%$ of all small intestinal carcinoids. ${ }^{1,3}$ They are seen with decreasing frequency from the first to the third part of the duodenum. ${ }^{1,2} \mathrm{~A} n$ association of duodenal carcinoids has been described with the Zollinger-Ellison syndrome and with multiple endocrine neoplasia, (MEN) type I.,2 Duodenal carcinoids excluding those arising from the periampullary region typically are slow growing with an indolent course. Periampullary carcinoids appear to have a distinctly different, more aggressive biology, with nodal involvement and metastases being unrelated to tumor size or mitotic activity. ${ }^{1-3}$ These tumors are hence considered separately from other duodenal carcinoid tumors. M ost of the duodenal carcinoids are clinically silent, but they may present with vague abdominal pain, vomiting as was the case with our patient. The may also present acutely with intestinal obstruction, bowel perforation, or rarely hemorrhage. ${ }^{1,2}$

Carcinoid tumors typically appear as firm, yellow nodules arising from the submucosa. EUS is an important modality to assess the depth of invasion and regional lymph nodal involvement. ${ }^{3,5-7}$ Due to its metastatic potential, it is imperative to perform EUS before deciding on the therapeutic strategy, appropriate case selection is important before planning endoscopic removal for duodenal carcinoids. ${ }^{3-7}$ Endoscopic treatment, such as EM R or EM D has been used sporadically as an alternative to conventional surgery. These techniques have their own spectrum of complications, the more sinister of them being perforation. ${ }^{7,8}$ In our technique of band ligation, the band has enough contractile force to squeeze the mucosal and submucosal layers, but is not strong enough to capture the muscularis propria layer thus avoiding this complication. ${ }^{2,8} \mathrm{~A}$ lso the use of the ubiquitous endoscopic band and a snare obviates the need for expensive accessories necessary for EM R/EM D of the duodenum. A part from our case report, there has only been one prior report of application of endoscopic bands with resection of duodenal carcinoids. ${ }^{8}$ The inherent rarity of this tumor and its endoscopic management makes it difficult to address the feasibility of endoscopic treatment. Features associated with an increased risk of metastasis as elucidated with Burke et al include involvement of the muscularis propria, size larger than $20 \mathrm{~mm}$, and the presence of mitotic figures. ${ }^{1}$ The metastatic rates are proportional to the size of the tumor: $8.3 \%$ with tumors smaller than $5 \mathrm{~mm}$ and $18.8 \%$ with those between 15.1 and $20 \mathrm{~mm}^{1-3}$ Therefore, if a nonperiampullary duodenal carcinoid smaller than $20 \mathrm{~mm}$ in diameter is diagnosed to be localized within the submucosal layer by EUS, endoscopic resection may be one of the preferred options for the treatment. ${ }^{7,8}$ Further, follow-up and larger studies are definitely required to address this technique's safely for long-term outcome of duodenal carcinoids.

\section{CONCLUSION}

Our report indicates that endoscopic banding and removal of small duodenal carcinoids located outside the periampullary region, with no EUS signs of invasion of the muscularis propria, is a safe, patient-friendly, adequate, effective and economical treatment. A Ithough larger studies are needed before endoscopic procedure can be incorporated into the standardized evidence based therapeutic protocol of these tumors. A report like ours is important to highlight this unique management for an uncommon tumor.

\section{REFERENCES}

1. Burke AP, Sobin LH, Federspiel BH, Shekitka KM, Helwig EB. Carcinoid tumors of the duodenum. A clinicopathologic study of 99 cases. Arch Pathol Lab M ed 1990;114:700-04.

2. Mullen J T, Wang $\mathrm{H}, \mathrm{Y}$ ao JC, L ee J H, Perrier ND, et al. Carcinoid tumors of the duodenum. Surgery 2005;138:971-78.

3. Y okoyama S, Takifuji K, Tani M, K awai M, Naka T, et al. Endoscopic resection of duodenal bulb neuroendocrine tumor larger than $10 \mathrm{~mm}$ in diameter. BM C Gastroenterol 2011; 11:67. 
4. Perng $C L$, Lin $H J, W$ ang $K$, Lai $C R$, Lee SD. Treatment of duodenal carcinoid by strip biopsy. J Clin Gastroenterol 1995;20(2):168-71.

5. Y oshikane H, Goto H, Niwa $Y, M$ atsui $M$, Ohashi $S$, et al. Endoscopic resection of small duodenal carcinoid tumors with strip biopsy technique. Gastrointest Endosc 1998;47(6):466-70.

6. $\mathrm{Y}$ amamoto $\mathrm{C}, \mathrm{A}$ oyagi $\mathrm{K}$, Suekane $\mathrm{H}$, Iida H, H izawa K, K uwano $Y$, et al. Carcinoid tumors of the duodenum: Report of three cases treated by endoscopic resection. Endoscopy 1997; 29(3):218-21.

7. Dalenbäck J, Havel G. Local endoscopic removal of duodenal carcinoid tumors. Endoscopy 2004;36(7):651-55.

8. Gomez V, Groce JR, Xaio SY, Bhutani M S, Raju GS. B and ligation resection of duodenal carcinoid (with video). Gastrointest Endosc 2007;66(2):397-98.

\section{ABOUT THE AUTHORS}

\section{Ashwin Rammohan (Corresponding Author)}

Sub Speciality Trainee, HPB and Liver Transplantation Surgery Institute of Surgical Gastroenterology and Liver Transplantation
Government Stanley M edical College and Hospital, Chennai, Tamil Nadu, India, e-mail: ashwinrammohan@gmail.com

\section{Sathyanesan Jeswanth}

Professor, Institute of Surgical Gastroenterology and Liver Transplantation, Centre for GI Bleed, Division of Hepatobiliary Pancreatic Diseases, Government Stanley Medical College and Hospital, Chennai, Tamil Nadu, India

\section{Perumal Senthil Kumar}

A ssistant Professor, Institute of Surgical Gastroenterology and Liver Transplantation, Centre for GI Bleed, Division of Hepatobiliary Pancreatic Diseases, Government Stanley Medical College and Hospital, Chennai, Tamil Nadu, India

\section{Palaniappan Ravichandran}

Professor and H ead, Institute of Surgical Gastroenterology and Liver Transplantation, Centre for GI Bleed, Division of Hepatobiliary Pancreatic Diseases, Government Stanley Medical College and Hospital, Chennai, Tamil Nadu, India 